\title{
Cell death, dysglycemia and myocardial infarction (Review)
}

\author{
XIAO-FANG TIAN ${ }^{1}$, MING-XIA CUI ${ }^{1}$, SHI-WEI YANG ${ }^{2}$, YU-JIE ZHOU ${ }^{2}$ and DA-YI HU ${ }^{3}$ \\ ${ }^{1}$ Key Laboratory of Preclinical Study for New Drugs of Gansu Province, Department of Pharmacology, School of Basic \\ Medical Sciences, Lanzhou University, Lanzhou, Gansu 730000; ${ }^{2} 12$ th Ward, Department of Cardiology, Beijing An Zhen \\ Hospital, Capital Medical University, Beijing Institute of Heart Lung and Blood Vessel Disease, The Key Laboratory of \\ Remodeling-Related Cardiovascular Disease, Ministry of Education, Beijing 100029; ${ }^{3}$ Department of Cardiology, \\ People's Hospital Affiliated to Peking University, Beijing 100044, P.R. China
}

Received December 8, 2012; Accepted February 11, 2013

DOI: $10.3892 /$ br.2013.67

\begin{abstract}
Dysglycemia (hyper- and hypoglycemia) has been associated with higher mortality among patients suffering from myocardial infarction (MI). Moreover, dysglycemia may induce cell death. Cell death (necrosis, apoptosis and autophagy) is a ubiquitous process that characterizes the course of several diseases, including MI, and occurs in diverse forms varying in mechanism, pattern and consequence. Therefore, cell death is a potential pathway through which dysglycemia affects the outcome of MI and it is essential to regulate myocardial cell death in the treatment of patients with MI caused by dysglycemia. In this review, we summarized the mechanisms of MI at the cellular level and the regulatory effects of dysglycemia on myocardial cell death. The ability to modulate myocardial cell death may be a promising target of new treatments aimed at limiting MI caused by dysglycemia. However, further research is required to elucidate the mechanisms underlying cell death regulation in MI caused by dysglycemia.
\end{abstract}

\section{Contents}

1. Introduction

2. Cell death

3. Classification and mechanisms of cell death

4. Cell death under the conditions of MI

5. Regulatory effects of dysglycemia on cell death

6. Conclusion

Correspondence to: Professor Yu-Jie Zhou or Professor Shi-Wei Yang, 12th Ward, Department of Cardiology, Key Laboratory of Remodeling-Related Cardiovascular Diseases, Beijing Anzhen Hospital, Capital Medical University, Ministry of Education, An Ding Men Wai, Chao Yang District, Beijing 100029, P.R. China

E-mail: azzyj12@163.com

E-mail: jackydang@163.com

Key words: cell death, hyperglycemia, hypoglycemia, myocardial infarction

\section{Introduction}

Cell death may be defined as an irreversible loss of plasma membrane integrity (1). Three types of cell death have been distinguished in mammalian cells according to morphological criteria: necrosis, apoptosis and autophagy. Cell death, either progressive or acute, is a hallmark characteristic of various cardiac diseases, including myocardial infarction (MI) (2). MI remains a leading cause of morbidity and mortality worldwide (3). MI occurs when myocardial ischemia (diminished blood supply to the heart) exceeds a critical threshold and overwhelms myocardial cellular repair mechanisms designed to maintain normal operating function and homeostasis (4). Dysglycemia (hyper- and hypoglycemia) is a strong predictor of mortality and increases the mortality risk among MI patients (5-7). Furthermore, previous studies reported a correlation between dysglycemia and cell death $(8,9)$. Therefore, we may hypothesize that cell death is a potential pathway through which dysglycemia affects the outcome of MI. It is imperative to achieve a full understanding of the mechanism underlying cardiomyocyte death and investigate the mechanisms by which dysglycemia affects the outcome of MI at the cellular lever.

\section{Cell death}

Cell death and cell proliferation are the two predominant physiological processes that regulate tissue homeostasis in the adult organism. They act to maintain cell numbers in tissues and organs at constant levels. Similar to cell proliferation, cell death is closely controlled in its activation and execution. Apart from biochemical processes occurring in the dying cell, execution ultimately involves the orderly removal of cell debris. Thus, cell death is an essential part of tissue renewal.

\section{Classification and mechanisms of cell death}

Necrosis. Type I cell death, most commonly known as necrosis, is often negatively perceived: death lacking the characteristics of programmed cell death and thus accidental and uncontrolled. However, previous studies demonstrated that necrosis is closely regulated. There are two main necrotic pathways: the death receptor pathway and mitochondrial pathway (10). 
The death receptor pathway of necrosis may be stimulated by tumor necrosis factor (TNF- $\alpha$ ), Fas ligand (FasL) and TNF-related apoptosis-inducing ligand. Programmed necrosis initiated by the ligation of TNF receptor (TNFR) 1, which is expressed in the majority of cell types and contains a cytoplasmic death domain (DD), as opposed to TNFR2, has been extensively characterized; thus, the research on death receptor-mediated necrosis focuses primarily on this pathway. Receptor-interacting protein (RIP) 1 consists of an N-terminal kinase domain, a RIP homotypic interaction motif and a C-terminal DD (11). Among these, DD mainly controls cell death. Therefore, TNFR1 is important due to its binding to the DD of RIP1 (12), which ultimately leads to cell necrosis. The mitochondrial pathway is an alternative pathway of necrosis. Anaerobic glycolysis is activated during ischemia to provide ATP, leading to the accumulation of $\mathrm{H}^{+}$and acidosis. $\mathrm{H}^{+}$is pumped out of the cell by the $\mathrm{Na}^{+} / \mathrm{H}^{+}$exchanger, which, in combination with the malfunctioning $\mathrm{Na}^{+} / \mathrm{Ca}_{2}{ }^{+}$exchanger operating in reverse mode, results in increased concentrations of cytoplasmic $\mathrm{Ca}_{2}{ }^{+}$. Increase in $\mathrm{Ca}_{2}{ }^{+}$in mitochondria induces $\mathrm{Ca}_{2}{ }^{+}$-dependent dehydrogenase activation, a decrease in $\mathrm{NADH}$ and electron flux through the electron transport chain, an increased production of reactive oxygen species (ROS) and a decrease in ATP levels. Moreover, ischemia results in ATP depletion and ROS generation, with the latter being further exacerbated during reperfusion. The long-lasting opening of the membrane permeability transition pore, which is regulated by cyclophilin D (CypD), is involved in this pathway $(2,13)$.

Apoptosis. Type II cell death, also known as apoptosis, has been well-characterized and the molecular events involved in apoptotic cell death are extensively comprehended. There are two major apoptotic signaling pathways: the extrinsic (also involving death receptors) and the intrinsic (mitochondria-mediated) pathways (14). The extrinsic pathway, being necrotic, involves death receptors. This pathway is also activated by death ligands, such as FasL or TNF- $\alpha$, which bind to cognate receptors on the plasma membrane with Fas or TNFR (15). However, numerous studies suggest that Fas, rather than TNFR, is the major mechanism underlying the activation of extrinsic apoptosis. In the extrinsic pathway, death receptor Fas is activated by FasL. This interaction leads to the recruitment of a DD (e.g., Fas-associated DD) and caspase- 8 activation, which in turn activates caspase-3. These events ultimately lead to cell apoptosis. By contrast, the intrinsic pathway is activated by a wide variety of apoptotic signals, including growth factor deprivation, hypoxia, oxidative stress and DNA damage (16). The mitochondrion is the primary organelle involved in the mediation of the intrinsic apoptotic pathway. Cytochrome $c$ $($ cyto- $c)$, which is released from mitochondria, is a key factor in this process. The regulation of cyto- $c$ release from mitochondria is modulated by the $\mathrm{Bcl}-2$ protein family. The $\mathrm{Bcl}-2$ proteins may be classified as anti-apoptotic (e.g., Bcl-2 and Bcl-xL) or pro-apoptotic (e.g., Bad, Bak and Bax) (14). In the intrinsic pathway, cyto- $c$ is induced by the pro-apoptotic Bax and Bak proteins. Subsequently, cyto- $c$ with apoptotic protein activating factor 1 (Apaf1) and caspase-9 form the apoptosome, with resulting activation of caspase-9. Caspase-9, in turn, activates caspase-3, leading to cell apoptosis. In addition to cyto- $c$, Smac/DIABLO, apoptosis inducing factor (AIF) and endonuclease $\mathrm{G}$ (Endo $\mathrm{G}$ ) are also released from the mitochondria. AIF and Endo G may directly induce apotosis, in contrast to Smac/DIABLO.

Although the extrinsic and intrinsic are two different pathways of apoptosis, they are intrinsically linked. One of the pro-apoptotic Bcl-2 family members, Bcl-2-interacting protein (Bid), may regulate the interaction between extrinsic and intrinsic pathways. Bid is usually located in the cytosol; however, when it is cleaved to 'truncated Bid' (tBid) by the activated caspase-8, Bid translocates to the mitochondria and regulates cyto- $c$ release (17).

Autophagy. Type III cell death, also known as autophagy, is a cell survival mechanism that involves the degradation and recycling of cytoplasmic components. Autophagy modulates cell death through excessive self-digestion and degradation of essential cellular constituents $(18,19)$. The regulation of autophagy is a complex process. Numerous signaling pathways, including nutrient signaling, insulin/growth factor pathways, energy sensing, stress response and pathogen infection, are crucial to the regulation of autophagy (20). Firstly, the central factor in nutrient signaling pathways is TOR/mTOR (21). TOR acts as an efficient gatekeeper in autophagy, on which it also exerts an inhibitory effect. In the presence of growth factors and abundant nutrients, it is the major inhibitory signal that shuts off autophagy. The mTOR pathway is regulated by the 5'-AMP-activated protein kinase (AMPK) (22). Glycogen synthase kinase-3 (GSK-3) is another regulator of the mTOR pathway in cardiac cells (23). Similar to AMPK, GSK-3 $\beta$ was also demonstrated to activate autophagy by inhibiting mTOR signaling (24). Secondly, both insulin/growth factor- and pathogen infection pathway-induced autophagy appears to be TOR-independent (25). Thirdly, the energy-sensing signaling pathway is also involved in autophagy regulation (26). Lum et al (27) reported that cellular ATP content may induce autophagy. In cultured cardiac myocytes, a decrease in ATP levels is often associated with an increase in AMP. AMPK, which activates autophagy by inhibiting mTOR signaling, is activated in response to elevations in the AMP/ATP ratio. Thus, autophagy may be upregulated by AMP through the activation of AMPK (28-30). Lastly, various extra- and intracellular stresses, which include ER stress $(31,32)$, hypoxia (33) and oxidative stress (34), also potently induce autophagy and it is important for organisms to adapt to or overcome unfavorable conditions. In the transcriptional regulation of autophagy, autophagy genes are regulated at the transcriptional level in response to stress. Forkhead box class O (FoxO) transcription factor was the first transcription factor demonstrated to be necessary and sufficient to induce autophagy in the Drosophila larval fat body (35). FoxO1 and FoxO3 are highly expressed in cardiac cells (36) and regulate autophagy by activating transcription of the Atg genes (37). Both FOX1 and FOX2 have been reported to induce expression of the Bcl-2/adenovirus E1B $19 \mathrm{kDa}$ protein-interacting protein 3 (BNIP3) $(38,39)$, which renders them potent inducers of autophagy.

Although the classification into different modes of death is useful, there is considerable overlap between the different mechanisms. For example, not all the characteristics of apoptosis are observed in all cell types and, in certain instances, the 
apoptotic cell may undergo secondary necrosis. In addition, previous studies indicated that apoptosis and autophagy may involve complementary pathways and that autophagic degeneration may be a part of apoptosis, at least in certain types of cells $(40,41)$.

Physiological and pathological roles of cell death. Although replacement of senescent cells with new cells requires programmed elimination of older, damaged or less functional tissue, an unexpected loss of cells accelerates growth of similar cells, in a healing process that is essential for functional homeostasis. However, abnormalities in the control of cell death contribute to a variety of diseases. Extensive retardation of cell death has been associated with human disease (42). For example, insufficient apoptosis may contribute to carcinogenesis. However, excessive cell death may be a component of the pathogenesis. These processes are significant in the pathogenesis of major diseases such as cancer, stroke, infection, inflammation and neurodegenerative disorders (43). In addition, a number of different types of cells undergo cell death in the cardiovascular system and may underlie several human heart diseases, including atherosclerosis, heart failure and MI (44). For example, the death of endothelial and vascular smooth muscle cells is involved in vessel injury and remodelling and in several vascular pathologies, such as atherosclerosis and aneurysm formation (45). Loss of cardiomyocytes is associated with ischemic and dilated cardiomyopathies, with the injury being attributed to ischemia/reperfusion (I/R), as well as with MI.

\section{Cell death under the conditions of MI}

Necrosis and MI. Necrosis, which was believed to be unregulated, was largely ignored in MI. Although cardiac myocyte necrosis is considered to be the major pathological lesion in acute MI (AMI), its significance in the pathogenesis was not formally evaluated until recently. A significant proportion of necrosis appears to be regulated and plays an important role in the pathogenesis of MI $(46,47)$. It has been demonstrated that the death receptor and mitochondrial pathways mediate cardiac myocyte necrosis and play a key role in MI. In the death receptor pathway, infarct size was markedly reduced in mice with Fas loss-of-function mutations $(48,49)$. In addition, genetic manipulation of the TNF- $\alpha$ signaling axis was also reported to be significant in $\mathrm{MI}$. When necrosis is activated by TNF- $\alpha$ with deletion of TNFR 1 and TNFR 2 , infarct size is exacerbated and coronary artery occlusion becomes permanent (50). Conversely, overexpression of TNF- $\alpha$ (at low levels) on a wild-type background restores myocardial damage (51). These studies suggested that the repressed death receptor pathway may limit MI during I/R. The role of the mitochondrial pathway in MI is also important. Deletion of CypD markedly decreases infarct size during I/R in vivo. Moreover, there are reports that necrosis occurring predominantly in the central area is more common between 6-24 h following MI (45). The extent of necrosis peaks at $24 \mathrm{~h}$ after reperfusion and remains constant thereafter $(52,53)$.

Apoptosis and MI. In the extrinsic pathway, mice that lacked Fas exhibited a decrease in cardiac myocyte apoptosis in models of doxorubicin toxicity, as well as marked reductions in infarct size following I/R (48). Cardiac myocyte-specific overexpression of Bcl-2 significantly reduced infarct size, cardiac myocyte apoptosis and cardiac dysfunction following I/R (54). Bax deficiency reduced infarct size and cardiac dysfunction following I/R and MI in mice (55). Taken together, these results suggested that the intrinsic apoptotic pathway also plays a central role in MI. Apoptosis has been demonstrated to be involved in the acute and chronic loss of cardiomyocytes in MI. Animal and human studies demonstrated that apoptosis was present in the border zone of the infarcted myocardium in the early phase (56). Permanent coronary occlusion induced maximum cardiac myocyte apoptosis at $4.5 \mathrm{~h}$ and reperfusion accelerated the timing of apoptosis, compared to permanent occlusion (57).

Autophagy and MI. Autophagy has also been detected in a variety of human cardiomyopathies, including ischemic heart disease, Danon disease, MI and heart failure (58). There is increasing evidence that autophagy, as a mechanism for the degradation of damaged long-lived proteins and organelles, plays an important role in the process of MI $(59,60)$. Further investigations are necessary, although current available reports indicate that autophagy exerts a predominantly cardioprotective effect during MI.

Of note, induction of autophagy by inhibition of mTOR with everolimus (RAD) prevents adverse left ventricular (LV) remodeling and limits infarct size following MI (61). Furthermore, autophagy promotes the survival of cardiomyocytes under extra- and intracellular stress (62). In addition, autophagy was shown to be cardioprotective (63). Brady et al (64) reported that preconditioning enhanced autophagy and inhibition of autophagy eliminated the cardioprotective effects of preconditioning. Moreover, autophagy may maintain LV function during starvation via functional FoxO (37). Induction of autophagy via these methods may be a novel therapeutic approach aimed at limiting infarct size, attenuating adverse LV remodeling, promoting cardiomyocyte survival and maintaining LV function.

\section{Regulatory effects of dysglycemia on cell death}

Regulatory effects of hyperglycemia on myocardial cell death. Numerous physiological studies $(8,65-69)$ have demonstrated that hyperglycemia exerts a direct detrimental effect on ischemic myocardium through several mechanisms. Hyperglycemia exerts a regulatory effect on cell death. Malhotra et al (65) demonstrated that hyperglycemia is a potent activating signal for cardiac protein kinase $\mathrm{C}$ (PKC) isozymes and induces the apoptosis program in cardiac myocytes. The PKC $\varepsilon$ translocation activator ( $\psi \varepsilon$ RACK) eliminated hyperglycemia-induced apoptosis, strongly suggesting a cardioprotective role for PKCe. Therefore, cardiac PKC isozymes modulate hyperglycemia-induced apoptosis and activation of cardiac PKCe protects adult rat ventricular myocytes (ARVM) against the hyperglycemia-induced death signal. Capes et al (66) reported that hyperglycemia induces ROS generation and apoptosis in cardiomyocytes, which contributes to diabetic cardiomyopathy. Rac1 is pivotal in hyperglycemia-induced apoptosis in cardiomyocytes. The 
role of Rac1 is mediated through NADPH oxidase activation and associated with mitochondrial ROS generation. Deficiency of Racl reduced hyperglycemia-induced mitochondrial ROS production in cardiac cells, significantly inhibited apoptosis and slightly improved myocardial function. Furthermore, a previous study demonstrated that the levels of Bcl-2 and Bax (well-known proteins involved in apoptosis) were evaluated under consistently high glucose levels in human umbilical vein endothelial cells (67). Whether this theory is applicable to myocardial cell apoptosis remains to be elucidated. These findings suggested that hyperglycemia exerts an effect on apoptosis in patients with MI. There are also numerous ongoing investigations regarding the role of hyperglycemia in the autophagy of cell death. Eguchi et al (8) reported that diabetic mice exhibited a reduced infarct size and reduced apoptosis $24 \mathrm{~h}$ after reperfusion, as indicated by TUNEL analysis in cardiac sections. This may be explained by increased autophagy through the AMPK-mTOR pathway detected in diabetic mice hearts. Diabetic mice exhibit a smaller infarct area post-MI, which may be the result of upregulated autophagy. Therefore, we may be able to limit infarct size caused by dysglycemia via the activation of autophagy. Furthermore, higher glucose levels in patients with AMI are associated with higher free fatty acid concentrations. The cellular autophagosome-lysosomal complexes in autophagy degrade a large number of free fatty acids in order to maintain the mitochondrial energy supply and improve cell survival through autophagy degradation within a certain range $(68,69)$ Thus, we may be able to protect cardiac myocytes against dysglycemia through the activation of autophagy. However, whether hyperglycemia is causally related to increased mortality due to a large infarct size and impaired LV function following AMI, or is simply an epiphenomenon of the severe disease conditions, remains to be elucidated; the mechanisms of the detrimental effect of hyperglycemia on the myocardium also require further investigation.

Regulatory effects of hypoglycemia on myocardial cell death. The pathophysiology of myocardial ischemia is an imbalance between myocardial oxygen demand and supply. Hypoglycemia may result in increased myocardial oxygen demand. In addition, hypoglycemia and coronary vasoconstriction limit the delivery of substrate (glucose and free fatty acids) to the myocardium, which further disrupts the balance between myocardial energy supply and demand (9). Therefore, the role of hypoglycemia in myocardial energy supply and demand at the cellular level is the focus of this review. Hypoglycemia also plays a role in the autophagy of cell death. Hypoglycemia causes a significant reduction in the levels of ATP. A decrease in ATP levels is accompanied by an increase in the AMP/ATP ratio, resulting in activation of the AMPK. Matsui et al (68) reported that glucose deprivation induced autophagy within a certain range via activation of AMPK in isolated cardiac myocytes. Decreased AMPK activity and subsequent reduction in cardiac autophagy are important events in the development of diabetic cardiomyopathy, as well as in MI. Therefore, we may be able to promote the survival of cardiomyocytes in the presence of dysglycemia by inducing autophagy.

\section{Conclusion}

Several studies have suggested that dysglycemia, including hyper- and hypoglycemia, is a strong predictor of mortality and significantly increases the mortality risk among MI patients. Furthermore, there are also studies (8,9,66-69) suggesting that dysglycemia induces myocardial cell death. Cell death (apoptosis, autophagy and necrosis) plays a significant role in MI. Thus, we hypothesize that cell death is a potential pathway through which dysglycemia influences the outcome of MI and that closely regulated myocardial cell death is essential in the treatment of patients with MI caused by dysglycemia. Due to the limited availability of clinical trial data suggesting that more efficient glucose control may improve patient outcomes following MI, in this review we aimed to summarize the mechanisms of MI at the cellular level, as well as the regulatory effects of dysglycemia on myocardial cell death. The ability to modulate myocardial cell death may be a promising therapeutic strategy in limiting MI caused by dysglycemia. However, the precise mechanisms of cell death in MI caused by dysglycemia, as well as the timing and manner of cell death modulation, remain to be elucidated.

\section{Acknowledgements}

The Beijing Heart and Metabolism Study was supported by a grant from the National Natural Science Foundation of China (no. 81100143), the Beijing Municipal Education Commission (no. KZ201110025031) and the Beijing Municipal Science and Technology Commission (no. Z0005190042811).

\section{References}

1. Kroemer G, Galluzzi L, Vandenabeele P, Abrams J, Alnemri ES, Baehrecke EH, Blagosklonny MV, El-Deiry WS, Golstein P, Green DR, Hengartner M, Knight RA, Kumar S, Lipton SA, Malorni W, Nunez G, Peter ME, Tschopp J, Yuan J, Piacentini M, Zhivotovsky B and Melino G: Classification of cell death: recommendations of the Nomenclature Committee on Cell Death 2009. Cell Death Differ 16: 3-11, 2009.

2. Whelan RS, Kaplinskiy V and Kitsis RN: Cell death in the pathogenesis of heart disease: mechanisms and significance. Annu Rev Physiol 72: 19-44, 2010.

3. Roger VL, Go AS, Lloyd-Jones DM, Benjamin EJ, Berry JD, Borden WB, Bravata DM, Dai S, Ford ES, Fox CS, Fullerton HJ, Gillespie C, Hailpern SM, Heit JA, Howard VJ, Kissela BM, Kittner SJ, Lackland DT, Lichtman JH, Lisabeth LD, Makuc DM, Marcus GM, Marelli A, Matchar DB, Moy CS, Mozaffarian D, Mussolino ME, Nichol G, Paynter NP, Soliman EZ, Sorlie PD, Sotoodehnia N, Turan TN, Virani SS, Wong ND, Woo D and Turner MB: Heart disease and stroke statistics - 2012 update: a report from the American Heart Association. Circulation 125: e2-e220, 2012.

4. Spindel $\mathrm{ON}$ and Berk BC: Redox redux: protecting the ischemic myocardium. J Clin Invest 122: 30-32, 2012.

5. Lee YA, Yoo JH, Kim JH, Lee SH, Kim JH, Lim HH, Kang MJ, Chung HR, Lee SY, Shin CH and Yang SW: Independent relationships of obesity and insulin resistance with serum proinsulin level in prepubertal children with normal glucose tolerance. Pediatr Diabetes 12: 235-241, 2011.

6. Yang SW, Zhou YJ, Nie XM, Liu YY, Du J, Hu DY, Jia DA, Gao F, Hu B, Fang Z, Han HY, Liu XL, Yan ZX, Wang JL, Hua Q, Shi YJ and Li HW: Effect of abnormal fasting plasma glucose level on all-cause mortality in older patients with acute myocardial infarction: results from the Beijing Elderly Acute Myocardial Infarction Study (BEAMIS). Mayo Clin Proc 86: 94-104, 2011. 
7. Yang SW, Zhou YJ, Liu YY, Hu DY, Shi YJ, Nie XM, Gao F, Hu B, Jia DA, Fang Z, Han HY, Wang JL, Hua Q and Li HW: Influence of abnormal fasting plasma glucose on left ventricular function in older patients with acute myocardial infarction. Angiology 63: 266-274, 2012.

8. Eguchi M, Kim YH, Kang KW, Shim CY, Jang Y, Dorval T, Kim KJ and Sweeney G: Ischemia-reperfusion injury leads to distinct temporal cardiac remodeling in normal versus diabetic mice. PLoS One 7: e30450, 2012.

9. Hsueh YC, Lee WH, Huang YY, et al: Hypoglycemia-induced non-ST segment-elevation myocardial infarction: an unusual complication of diabetes mellitus. Acta Cardiol Sin 28: 148-151, 2012.

10. Lim SY, Davidson SM, Mocanu MM, Yellon DM and Smith CC: The cardioprotective effect of necrostatin requires the cyclophilin-D component of the mitochondrial permeability transition pore. Cardiovasc Drugs Ther 21: 467-469, 2007.

11. Festjens N, Vanden BT, Cornelis S and Vandenabeele P: RIP1, a kinase on the crossroads of a cell's decision to live or die. Cell Death Differ 14: 400-410, 2007.

12. Lavrik IN, Mock T, Golks A, Hoffmann JC, Baumann S and Krammer PH: CD95 stimulation results in the formation of a novel death effector domain protein-containing complex. J Biol Chem 283: 26401-26408, 2008.

13. Kitsis RN and Molkentin JD: Apoptotic cell death 'Nixed' by an ER-mitochondrial necrotic pathway. Proc Natl Acad Sci USA 107: 9031-9032, 2010.

14. Youle RJ and Strasser A: The BCL-2 protein family: opposing activities that mediate cell death. Nat Rev Mol Cell Biol 9: 47-59, 2008.

15. Ashkenazi A and Dixit VM: Death receptors: signaling and modulation. Science 281: 1305-1308, 1998.

16. Kaufmann T, Tai L, Ekert PG, Huang DC, Norris F, Lindemann RK, Johnstone RW, Dixit VM and Strasser A: The $\mathrm{BH} 3$-only protein bid is dispensable for DNA damage- and replicative stress-induced apoptosis or cell-cycle arrest. Cell 129: 423-433, 2007

17. Li H, Zhu H, Xu CJ and Yuan J: Cleavage of BID by caspase 8 mediates the mitochondrial damage in the Fas pathway of apoptosis. Cell 94: 491-501, 1998.

18. Levine B and Yuan J: Autophagy in cell death: an innocent convict. J Clin Invest 115: 2679-2688, 2005.

19. Shimizu S, Kanaseki T, Mizushima N, Mizuta T, ArakawaKobayashi S, Thompson CB and Tsujimoto Y: Role of Bcl-2 family proteins in a non-apoptotic programmed cell death dependent on autophagy genes. Nat Cell Biol 6: 1221-1228, 2004

20. He C and Klionsky DJ: Regulation mechanisms and signaling pathways of autophagy. Annu Rev Genet 43: 67-93, 2009.

21. Yang YP, Liang ZQ, Gu ZL and Qin ZH: Molecular mechanism and regulation of autophagy. Acta Pharmacol Sin 26: 1421-1434, 2005.

22. Gwinn DM, Shackelford DB, Egan DF, Mihaylova MM, Mery A, Vasquez DS, Turk BE and Shaw RJ: AMPK phosphorylation of raptor mediates a metabolic checkpoint. Mol Cell 30: 214-226, 2008.

23. Hirotani S, Zhai P, Tomita H, Galeotti J, Marquez JP, Gao S, Hong C, Yatani A, Avila J and Sadoshima J: Inhibition of glycogen synthase kinase 3beta during heart failure is protective. Circ Res 101: 1164-1174, 2007.

24. Buller CL, Loberg RD, Fan MH, Zhu Q, Park JL, Vesely E, Inoki K, Guan KL and Brosius FC III: A GSK-3/TSC2/mTOR pathway regulates glucose uptake and GLUT1 glucose transporter expression. Am J Physiol Cell Physiol 295: C836-C843, 2008.

25. Wang Y, Weiss LM and Orlofsky A: Host cell autophagy is induced by Toxoplasma gondii and contributes to parasite growth. J Biol Chem 284: 1694-1701, 2009.

26. Robaglia C, Thomas M and Meyer C: Sensing nutrient and energy status by SnRK1 and TOR kinases. Curr Opin Plant Biol 15: 301-307, 2012.

27. Lum JJ, DeBerardinis RJ and Thompson CB: Autophagy in metazoans: cell survival in the land of plenty. Nat Rev Mol Cell Biol 6: 439-448, 2005.

28. Costa R, Morrison A, Wang J, Manithody C, Li J and Rezaie AR: Activated protein $\mathrm{C}$ modulates cardiac metabolism and augments autophagy in the ischemic heart. J Thromb Haemost 10: 1736-1744, 2012

29. Hardie DG: AMP-activated/SNF1 protein kinases: conserved guardians of cellular energy. Nat Rev Mol Cell Biol 8: 774-785, 2007.
30. Arad M, Seidman CE and Seidman JG: AMP-activated protein kinase in the heart: role during health and disease. Circ Res 100: 474-488, 2007.

31. Bernales S, McDonald KL and Walter P: Autophagy counterbalances endoplasmic reticulum expansion during the unfolded protein response. PLoS Biol 4: e423, 2006.

32. Sakaki K, Wu J and Kaufman RJ: Protein kinase Ctheta is required for autophagy in response to stress in the endoplasmic reticulum. J Biol Chem 283: 15370-15380, 2008.

33. Chen Y, McMillan-Ward E, Kong J, Israels SJ and Gibson SB Mitochondrial electron-transport-chain inhibitors of complexes I and II induce autophagic cell death mediated by reactive oxygen species. J Cell Sci 120: 4155-4166, 2007.

34. Chen Y, McMillan-Ward E, Kong J, Israels SJ and Gibson SB: Oxidative stress induces autophagic cell death independent of apoptosis in transformed and cancer cells. Cell Death Differ 15: 171-182, 2008.

35. Juhasz G, Puskas LG, Komonyi O, Erdi B, Maroy P, Neufeld TP and Sass M: Gene expression profiling identifies FKBP39 as an inhibitor of autophagy in larval Drosophila fat body. Cell Death Differ 14: 1181-1190, 2007.

36. Hannenhalli S, Putt ME, Gilmore JM, Wang J, Parmacek MS, Epstein JA, Morrisey EE, Margulies KB and Cappola TP: Transcriptional genomics associates FOX transcription factors with human heart failure. Circulation 114: 1269-1276, 2006.

37. Sengupta A, Molkentin JD, Paik JH, DePinho RA and Yutzey KE: FoxO transcription factors promote cardiomyocyte survival upon induction of oxidative stress. J Biol Chem 286 : 7468-7478, 2011.

38. Mammucari C, Milan G, Romanello V, Masiero E, Rudolf R, Del Piccolo P, Burden SJ, Di Lisi R, Sandri C, Zhao J, Goldberg AL, Schiaffino S and Sandri M: FoxO3 controls autophagy in skeletal muscle in vivo. Cell Metab 6: 458-471, 2007.

39. Xu P, Das M, Reilly J and Davis RJ: JNK regulates FoxO-dependent autophagy in neurons. Genes Dev 25: 310-322, 2011.

40. Pattingre S, Tassa A, Qu X, Garuti R, Liang XH, Mizushima N, Packer M, Schneider MD and Levine B: Bcl-2 antiapoptotic proteins inhibit Beclin 1-dependent autophagy. Cell 122: 927-939, 2005.

41. Sugishita Y, Watanabe M and Fisher SA: The development of the embryonic outflow tract provides novel insights into cardiac differentiation and remodeling. Trends Cardiovasc Med 14: 235-241, 2004

42. Thompson CB: Apoptosis in the pathogenesis and treatment of disease. Science 267: 1456-1462, 1995.

43. Lorenzen JM, David S, Richter A, de Groot K, Kielstein JT, Haller H, Thum T and Fliser D: TLR- $4^{+}$peripheral blood monocytes and cardiovascular events in patients with chronic kidney disease - a prospective follow-up study. Nephrol Dial Transplant 26: 1421-1424, 2011

44. Lockshin RA and Zakeri Z: Cell death in health and disease. J Cell Mol Med 11: 1214-1224, 2007.

45. Clarke M, Bennett M and Littlewood T: Cell death in the cardiovascular system. Heart 93: 659-664, 2007.

46. Baines CP, Kaiser RA, Purcell NH, Blair NS, Osinska H, Hambleton MA, Brunskill EW, Sayen MR, Gottlieb RA, Dorn GW, Robbins J and Molkentin JD: Loss of cyclophilin D reveals a critical role for mitochondrial permeability transition in cell death. Nature 434: 658-662, 2005.

47. Nakagawa T, Shimizu S, Watanabe T, Yamaguchi O, Otsu K, Yamagata $\mathrm{H}$, Inohara $\mathrm{H}$, Kubo $\mathrm{T}$ and Tsujimoto $\mathrm{Y}$ : Cyclophilin D-dependent mitochondrial permeability transition regulates some necrotic but not apoptotic cell death. Nature 434: $652-658,2005$

48. Lee P, Sata M, Lefer DJ, Factor SM, Walsh K and Kitsis RN: Fas pathway is a critical mediator of cardiac myocyte death and MI during ischemia-reperfusion in vivo. Am J Physiol Heart Circ Physiol 284: H456-H463, 2003

49. Jeremias I, Kupatt C, Martin-Villalba A, Habazettl H, Schenkel J, Boekstegers P and Debatin KM: Involvement of CD95/Apo1/Fas in cell death after myocardial ischemia. Circulation 102: 915-920, 2000.

50. Kurrelmeyer KM, Michael LH, Baumgarten G, Taffet GE, Peschon JJ, Sivasubramanian N, Entman ML and Mann DL: Endogenous tumor necrosis factor protects the adult cardiac myocyte against ischemic-induced apoptosis in a murine model of acute myocardial infarction. Proc Natl Acad Sci USA 97: 5456-5461, 2000 
51. Burchfield JS, Dong JW, Sakata Y, Gao F, Tzeng HP, Topkara VK, Entman ML, Sivasubramanian N and Mann DL: The cytoprotective effects of tumor necrosis factor are conveyed through tumor necrosis factor receptor-associated factor 2 in the heart. Circ Heart Fail 3: 157-164, 2010.

52. Kajstura J, Cheng W, Reiss K, Clark WA, Sonnenblick EH, Krajewski S, Reed JC, Olivetti G and Anversa P: Apoptotic and necrotic myocyte cell deaths are independent contributing variables of infarct size in rats. Lab Invest 74: 86-107, 1996.

53. Zhao ZQ and Vinten-Johansen J: Myocardial apoptosis and ischemic preconditioning. Cardiovasc Res 55: 438-455, 2002

54. Chen Z, Chua CC, Ho YS, Hamdy RC and Chua BH: Overexpression of $\mathrm{Bcl}-2$ attenuates apoptosis and protects against myocardial I/R injury in transgenic mice. Am J Physiol Heart Circ Physiol 280: H2313-H2320, 2001.

55. Hochhauser E, Cheporko Y, Yasovich N, Pinchas L, Offen D, Barhum Y, Pannet H, Tobar A, Vidne BA and Birk E: Bax deficiency reduces infarct size and improves long-term function after myocardial infarction. Cell Biochem Biophys 47: 11-20, 2007.

56. Olivetti G, Quaini F, Sala R, Lagrasta C, Corradi D, Bonacina E, Gambert SR, Cigola E and Anversa P: Acute myocardial infarction in humans is associated with activation of programmed myocyte cell death in the surviving portion of the heart. J Mol Cell Cardiol 28: 2005-2016, 1996.

57. Fliss H and Gattinger D: Apoptosis in ischemic and reperfused rat myocardium. Circ Res 79: 949-956, 1996.

58. Kirshenbaum LA: Regulation of autophagy in the heart in health and disease. J Cardiovasc Pharmacol 60: 109, 2012.

59. Nishida K, Kyoi S, Yamaguchi O, Sadoshima J and Otsu K: The role of autophagy in the heart. Cell Death Differ 16: 31-38, 2009.

60. Tucka J, Bennett $M$ and Littlewood T: Cell death and survival signalling in the cardiovascular system. Front Biosci 17: 248-261, 2012.

61. Nakai A, Yamaguchi O, Takeda T, Higuchi Y, Hikoso S, Taniike M, Omiya S, Mizote I, Matsumura Y, Asahi M, Nishida K, Hori M, Mizushima N and Otsu K: The role of autophagy in cardiomyocytes in the basal state and in response to hemodynamic stress. Nat Med 13: 619-624, 2007.
62. Sciarretta S, Zhai P, Shao D, Maejima Y, Robbins J, Volpe M, Condorelli G and Sadoshima J: Rheb is a critical regulator of autophagy during myocardial ischemia: pathophysiological implications in obesity and metabolic syndrome. Circulation 125: 1134-1146, 2012.

63. Gustafsson AB and Gottlieb RA: Autophagy in ischemic heart disease. Circ Res 104: 150-158, 2009.

64. Brady NR, Hamacher-Brady A, Yuan H and Gottlieb RA: The autophagic response to nutrient deprivation in the hl-1 cardiac myocyte is modulated by $\mathrm{Bcl}-2$ and sarco/endoplasmic reticulum calcium stores. FEBS J 274: 3184-3197, 2007.

65. Malhotra A, Kang BP, Hashmi S and Meggs LG: PKCepsilon inhibits the hyperglycemia-induced apoptosis signal in adult rat ventricular myocytes. Mol Cell Biochem 268: 169-173, 2005.

66. Capes SE, Hunt D, Malmberg K and Gerstein HC: Stress hyperglycaemia and increased risk of death after myocardial infarction in patients with and without diabetes: a systematic overview. Lancet 355: 773-778, 2000.

67. Risso A, Mercuri F, Quagliaro L, Damante G and Ceriello A: Intermittent high glucose enhances apoptosis in human umbilical vein endothelial cells in culture. Am J Physiol Endocrinol Metab 281: E924-E930, 2001.

68. Matsui Y, Takagi H, Qu X, Abdellatif M, Sakoda H, Asano T, Levine B and Sadoshima J: Distinct roles of autophagy in the heart during ischemia and reperfusion: roles of AMP-activated protein kinase and Beclin 1 in mediating autophagy. Circ Res 100: 914-922, 2007.

69. Zhang JL, Lu JK, Chen D, Cai Q, Li TX, Wu LS and Wu XS Myocardial autophagy variation during acute myocardial infarction in rats: the effects of carvedilol. Chin Med J (Engl) 122: 2372-2379, 2009 